\title{
Thermoneutral Regulation and Acute Injury: Implications for Acute Kidney Injury
}

\author{
Lisa M. Curtis Rohan Balkawade \\ Division of Nephrology, Department of Medicine, University of Alabama, Birmingham, AL, USA
}

\section{Keywords}

Facultative thermoneutrality - Sex differences $\cdot$ Acute kidney injury · Mitochondria

\begin{abstract}
Acute kidney injury (AKI) has demonstrated sex differences as illustrated in clinical and preclinical studies. In most cases, females show a significant resistance to AKI as manifested by renal indicators of injury, and thus much of the literature is derived from studies exclusively in males. Thermoneutral housing alters sex differences in acute injury of the liver, but has not been studied in the kidney. Thermoneutrality, the ambient temperature at which additional energy is not needed to maintain core body temperature, is regulated by mechanisms residing in mitochondria. Importantly, mitochondrial function plays an important role in induction and recovery of $\mathrm{AKI}$. Mechanisms that regulate thermoneutrality include uncoupling proteins (UCPs) and one of its upstream regulators peroxisome proliferator-activated receptor gamma coactivator-1a (PGC-1a). PGC-1a has been extensively studied in AKI in males. UCP-2, a UCP expressed in the kidney, has been minimally studied in AKI in males. Expression of other UCPs in the kidney has not been well defined. No studies of either PGC-1 a or UCPs have interrogated for a sex difference nor have they been investigated at thermoneutrality in the kidney. In this brief review, pathways of importance in thermoneutrality are described and related to path-
\end{abstract}

ways of importance in modulating susceptibility to AKI. Clarity in the understanding of the impact of thermoneutrality on AKI in altering susceptibility in females may expand our understanding of the critical role of mitochondrial function in this setting. Unique utilization of mitochondrial-based molecular pathways in females may then inform potential therapies.

(c) 2021 S. Karger AG, Basel

\section{Introduction}

Acute kidney injury (AKI) is a clinically challenging problem due to an absence of intervention beyond dialysis, which is mainly supportive. Women demonstrate a reduced incidence of AKI in most settings with the exception of AKI resulting from cardiac surgery [1]. Preclinical modeling of AKI similarly shows a resistance to AKI in females [2]. Ischemia-reperfusion injury models consistently require longer ischemia times in females than in males to illicit similar loss of kidney function.

Contribution from the AKI and CRRT 2021 Symposium at the 26th International Conference on Advances in Critical Care Nephrology, A Virtual/Hybrid Event from San Diego, CA, USA, February 28-March 5, 2021. This symposium was supported in part by the NIDDK funded University of Alabama at Birmingham-University of California San Diego O'Brien Center for Acute Kidney Injury Research (P30DK079337). karger@karger.com

www.karger.com/nef

Karger $\stackrel{2}{*}$
(C) 2021 S. Karger AG, Basel

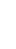

Correspondence to:

Lisa M. Curtis, lisacurtis@uabmc.edu 
Cisplatin-induced AKI has been used infrequently in females, and those studies that have used females, are discordant in demonstrating differing susceptibility by sex. Importantly, these latter studies have used different dosing schemes.

Sex differences seen in acute liver injury are modulated by changes in ambient housing temperatures of rodents [3]. Immune cell fluxes and various pathways involved in inflammation and metabolism are modulated with changes in ambient temperature. Immune cells, including monocytes and T cells, show differential mobilization or activation in response to different ambient housing temperatures [4]. Graft versus host disease in bone marrow transplantation in mice is exacerbated by thermoneutral housing. Thermoneutral housing alters outcomes in inflammation and immune cell responses in the liver, spleen, and vasculature [3-5]. Thermoneutral conditions have not been studied for AKI, but the pathways involved in thermoneutral regulation have relevance in AKI (Fig. 1) and may have importance in modulating susceptibility to AKI in females. Clarity in understanding the mechanisms that modulate sex-based susceptibility to AKI may provide insight into novel potential therapeutic pathways.

\section{Main Text}

Core body temperature is controlled by thyroid hormone through its action on brown adipose tissue (BAT), and thus this tissue has been extensively studied in thermogenesis. Two types of thermogenesis occur, obligatory and facultative. Obligatory thermogenesis generates heat at a baseline metabolism. Thermoneutrality is defined as the ambient temperature at which additional energy beyond that generated by obligatory thermogenesis is not required to maintain core body temperature, and thus metabolism is at a nadir. Facultative thermogenesis (FT) occurs in settings wherein obligatory thermogenesis is not sufficient to maintain core body temperature, that is, below the thermoneutral zone [6]. Preclinical models are conducted on rodents that are housed at vivaria temperatures $\left(23-27^{\circ} \mathrm{C}\right)$ that are set for human comfort. Thermoneutral temperature for rodents is approximately $30^{\circ} \mathrm{C}$. Thus, typical vivaria temperatures represent a chronic "cold stress." In cold stress, organisms initiate FT with the accompanying changes to metabolism to maintain core body temperature [6].

At baseline metabolism, the electron transport chain in the mitochondria generates energy by creating a $\mathrm{H}^{+}$

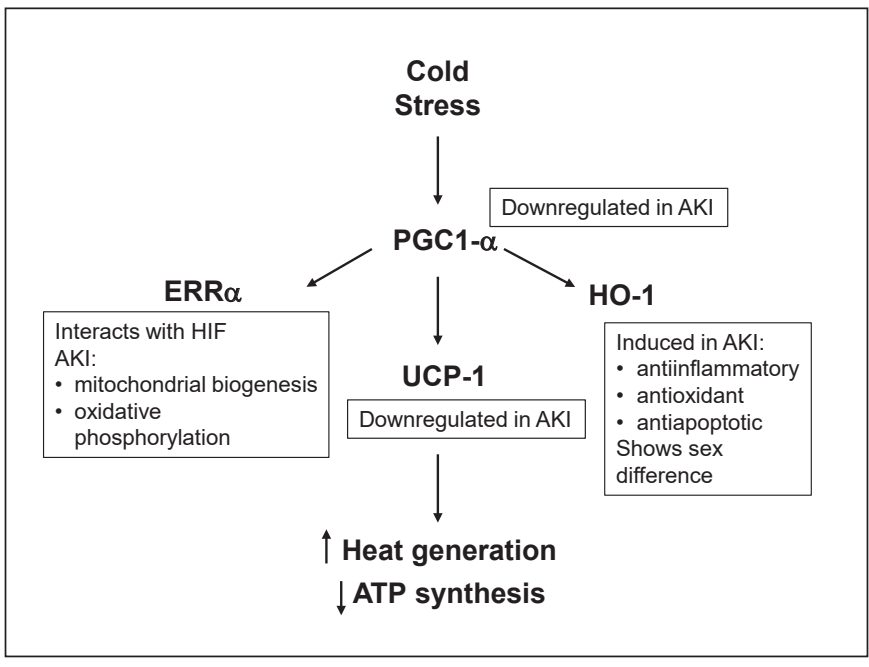

Fig. 1. Correlation between the thermogenic pathway and effects seen in AKI. Canonical thermogenic mechanisms seen in brown adipose tissue include cold stress-activation of the master regulator, PGC-1 $\alpha$. Subsequent activation of UCPs, and specifically UCP-1 in brown fat, initiates a proton leak across the mitochondrial membrane which results in less ATP production and the generation of heat. Downstream of PGC-1a, isoforms of ERR as well as HO-1 are modulated. In AKI, PGC- $1 \alpha$ is downregulated. ERR $\alpha$ has been shown to interact with HIF, which is induced under hypoxic conditions including that seen in AKI. ERRa also has been shown in AKI to modulate mitochondrial biogenesis and oxidative phosphorylation. HO-1 is induced in AKI and shows a sex-based temporal effect on its expression. It has anti-inflammatory, antioxidant, and antiapoptotic effects which are thought to confer protection in AKI. PGC-1 $\alpha$, peroxisome proliferator-activated receptor gamma coactivator-1 $\alpha$; UCPs, uncoupling proteins; ERR, estrogen-related receptor; HO-1, heme oxygenase-1; HIF, hypoxia-inducible factor.

gradient across the inner mitochondrial membrane which drives ATP production via the enzyme ATP synthase. However, the coupling of oxidation to ATP is not perfect, and some portion of the energy released is dissipated as heat. The uncoupling protein (UCP), which resides on the inner membrane, provides an alternative, low-impedance route for the protons to move across the inner membrane thereby collapsing the gradient, bypassing the ATP synthase, and producing heat [4]. FT relies on this latter mechanism for heat generation. Recent studies have begun to examine sex differences in rodents housed at thermoneutrality, and data suggest that housing female rodents at thermoneutrality may lessen the resistance to acute liver injury in females [3].

The kidney has the second highest mitochondrial content and resting metabolic rate after the heart. Mitochon- 
drial energetics are noted consistently as a contributor to the outcomes of AKI [7], and sex differences are apparent in mitochondrial function (reviewed in [8]). Mitochondrial transplantation via intra-arterial injection in female Yorkshire pigs reduced measures of renal injury in a model of ischemia-reperfusion injury [9], but was not studied in males.

In general, experimental modulation of sex differences focuses on the role of gonadal hormones through gonadectomy or pharmacological manipulation of hormone levels. Loss of estrogen signaling via pharmacological inhibition of estrogen receptors or surgical removal of gonads modulates susceptibility to AKI [10]. Estrogen receptors have been shown to localize to mitochondria in the heart [11], and estrogen receptor activation has been shown to modulate pathways of mitochondrial $\beta$-oxidation in the kidney $[12,13]$. Estrogen acting via the $\mathrm{ER} \alpha$ receptor in the hypothalamus has been shown to modulate thermogenesis [14]. Estrogen-related receptors (ERR) share homology with estrogen receptors and some downstream targets. ERR $a$ may be induced by estrogen [15], but a role for ERRa in AKI in females has not been studied. ERRa expression in males is protective in cisplatin-induced AKI, reducing damage in renal tubules and mitochondria as well as preserving renal function [13]. ERRa may interact with hypoxia-inducible factor (HIF) [16], which is upregulated in AKI, and induce HIF-dependent transcription. Adrenergic stimulation of ERRs in BAT shows an acute response to thermogenesis while mice lacking adipose ERRs have reduced oxidative and thermogenic capacity and rapidly become hypothermic when exposed to cold [17]. Furthermore, subcutaneous and intracerebroventricular administration of estradiol in OVX rats increased the mRNA expression or protein content of thermogenic markers, such as UCP-1 and peroxisome proliferator-activated receptor gamma coactivator-1 $\alpha$ (PGC1 $\alpha)$ in BAT [14]. ERR $\alpha$ and ERR $\gamma$ may be induced by PGC-1a [18] and modulate mitochondrial biogenesis and oxidative phosphorylation [13, 19].

PGC- $1 \alpha$ is a master regulator of several pathways that are important in mitochondrial function, including mitochondrial biogenesis, as well as directing the thermogenic response, and may be important in AKI [20]. Downregulation of PGC-1 $\alpha$ occurs in a model of cisplatin-induced AKI in males [21]. PGC-1a downregulation has also been shown to exacerbate septic AKI [22]. Overexpression of PGC-1 $\alpha$ is protective through alteration of mitophagy, a process of recycling mitochondria [23]. Higher levels of PGC-1a have been as-

Thermoneutrality and AKI sociated with reduced delayed graft function in patients [24].

Downstream targets of PGC-1 $\alpha$ may also have importance for modulating AKI. PGC-1 1 regulates expression of UCPs [25]. Induction of UCPs during thermoregulation in brown fat shows a sex difference [26]. UCP expression in the kidney includes UCP-2 [27] and may also include UCP-1 [28]. UCP-3 expression is upregulated in renal cell carcinoma [29] which may reflect a potential for modulation in the kidney in other circumstances. Other UCPs have not been described in the kidney. UCP1 is the canonical UCP found in BAT (Fig. 1). In mouse models of bilateral renal ischemia-reperfusion injury and cisplatin-induced kidney injury, downregulation of UCP-1 was noted [28]. UCP-2 may have a direct role in mitochondrial uncoupling [30], but also may be increased in response to hypoxia [31]. Modulation of UCP2 in the kidney has not been extensively studied, but in a mouse model of bilateral IRI, UCP-2 upregulation alleviates kidney injury while proximal tubule deletion of UCP-2 exacerbates the renal injury [32]. PGC-1a also may modulate expression of heme oxygenase-1 (HO-1) [33]. PGC-1 $\alpha$ has been shown to interact with nuclear factor erythroid 2-related factor $2[34,35]$, which can activate $\mathrm{HO}-1$ expression. $\mathrm{HO}-1$ is an inducible stress enzyme which is expressed on the endoplasmic reticulum membrane. It confers protection from AKI through its enzymatic activity degrading heme moieties. Additionally, the effects of HO- 1 on gene transcription to produce anti-inflammatory, antioxidant, and antiapoptotic effects are provided by an induced cleaved form, which is no longer membrane bound. The expression of the cleaved form shows a temporal divergence by sex, with early elevation after cisplatin-induced AKI followed by a decline to baseline levels sooner in females than in males [36].

\section{Conclusion}

While these critical pathways altered with thermogenesis have not been explored in AKI, the confluence of evidence in other organ systems suggests that sex differences in AKI may be modulated by thermoneutral housing of rodents. These pathways that show regulation by estrogen, and which in males can alter the measures of injury in AKI, may be important in driving resistance to AKI in females. Future studies to better understand these mechanisms in AKI in males and females are needed. Humans modulate their "ambient" temper- 
ature via wearing clothes and residing indoors with temperature control. The actions of these critical pathways may nonetheless alter injury in AKI in patients and may represent untapped potential for novel therapies for AKI.

\section{Conflict of Interest Statement}

The authors have no conflicts of interest to declare.

\section{Funding Sources}

L.M.C. was supported by the UAB-UCSD O'Brien Center for Acute Kidney Research (NIH/NIDDK P30 DK079337) and a Sex Differences Supplement to the same.

\section{Author Contributions}

L.M.C. composed the manuscript. R.B. and L.M.C. revised the manuscript.

\section{References}

1 Neugarten J, Golestaneh L. Female sex reduces the risk of hospital-associated acute kidney injury: a meta-analysis. BMC Nephrol. 2018; 19:314.

2 Aufhauser DD Jr, Wang Z, Murken DR, Bhatti TR, Wang Y, Ge G, et al. Improved renal ischemia tolerance in females influences kidney transplantation outcomes. J Clin Invest. 2016; 126:1968-77.

3 Giles DA, Moreno-Fernandez ME, Stankiewicz TE, Graspeuntner S, Cappelletti M, Wu $\mathrm{D}$, et al. Thermoneutral housing exacerbates nonalcoholic fatty liver disease in mice and allows for sex-independent disease modeling. Nat Med. 2017;23:829.

4 Kokolus K, Spangler H, Povinelli B, Farren M, Lee K, Repasky E. Stressful presentations: mild cold stress in laboratory mice influences phenotype of dendritic cells in naïve and tumor-bearing mice. Front Immunol. 2014;5: 23

5 Giles DA, Ramkhelawon B, Donelan EM, Stankiewicz TE, Hutchison SB, Mukherjee R, et al. Modulation of ambient temperature promotes inflammation and initiates atherosclerosis in wild type c57bl/6 mice. Mol Metab. 2016;5:1121-30.

6 Silva JE. Thermogenic mechanisms and their hormonal regulation. Physiol Rev. 2006;86: 435-64.

7 Lan R, Geng H, Singha PK, Saikumar P, Bottinger EP, Weinberg JM, et al. Mitochondrial pathology and glycolytic shift during proximal tubule atrophy after ischemic aki. J Am Soc Nephrol. 2016;27:3356-67.

8 Sultanova R, Schibalski R, Yankelevich IA, Stadler K, Ilatovskaya DV. Sex differences in renal mitochondrial function: a hormonegous opportunity for research. Am J Physiol Renal Physiol. 2020;319:F1117-24.

9 Doulamis IP, Guariento A, Duignan T, Kido T, Orfany A, Saeed MY, et al. Mitochondrial transplantation by intra-arterial injection for acute kidney injury. Am J Physiol Renal Physiol. 2020;319:F403-13.

10 Kang KP, Lee JE, Lee AS, Jung YJ, Kim D, Lee $S$, et al. Effect of gender differences on the regulation of renal ischemia-reperfusion-induced inflammation in mice. Mol Med Rep. 2014;9:2061-8.
11 Yang SH, Liu R, Perez EJ, Wen Y, Stevens SM $\mathrm{Jr}$, Valencia T, et al. Mitochondrial localization of estrogen receptor beta. Proc Natl Acad Sci U S A. 2004;101:4130-5.

12 Nishi Y, Satoh M, Nagasu H, Kadoya H, Ihoriya C, Kidokoro K, et al. Selective estrogen receptor modulation attenuates proteinuria-induced renal tubular damage by modulating mitochondrial oxidative status. Kidney Int. 2013;83:662-73.

13 Tsushida K, Tanabe K, Masuda K, Tanimura $\mathrm{S}$, Miyake H, Arata Y, et al. Estrogen-related receptor $\alpha$ is essential for maintaining mitochondrial integrity in cisplatin-induced acute kidney injury. Biochem Biophys Res Commun. 2018;498:918-24.

14 Martínez de Morentin Pablo B, GonzálezGarcía I, Martins L, Lage R, Fernández-Mallo $\mathrm{D}$, Martínez-Sánchez N, et al. Estradiol regulates brown adipose tissue thermogenesis via hypothalamic ampk. Cell Metab. 2014;20:4153.

15 Liu D, Zhang Z, Gladwell W, Teng CT. Estrogen stimulates estrogen-related receptor alpha gene expression through conserved hormone response elements. Endocrinology. 2003;144:4894-904.

16 Ao A, Wang H, Kamarajugadda S, Lu J. Involvement of estrogen-related receptors in transcriptional response to hypoxia and growth of solid tumors. Proc Natl Acad Sci U S A. 2008; 105:7821-6.

17 Brown EL, Hazen BC, Eury E, Wattez JS, Gantner ML, Albert V, et al. Estrogen-related receptors mediate the adaptive response of brown adipose tissue to adrenergic stimulation. iScience. 2018;2:221-37.

18 Schreiber SN, Knutti D, Brogli K, Uhlmann T, Kralli A. The transcriptional coactivator pgc1 regulates the expression and activity of the orphan nuclear receptor estrogen-related receptor alpha (erralpha). J Biol Chem. 2003; 278:9013-8.

19 Schreiber SN, Emter R, Hock MB, Knutti D, Cardenas J, Podvinec M, et al. The estrogenrelated receptor alpha (erralpha) functions in ppargamma coactivator 1alpha (pgc-1alpha)induced mitochondrial biogenesis. Proc Natl Acad Sci U S A. 2004;101:6472-7.
20 Lynch MR, Tran MT, Parikh SM. PGC1a in the kidney. Am J Physiol Renal Physiol. 2018; 314(1):F1-8.

21 Portilla D, Dai G, McClure T, Bates L, Kurten $\mathrm{R}$, Megyesi J, et al. Alterations of pparalpha and its coactivator pgc-1 in cisplatin-induced acute renal failure. Kidney Int. 2002;62:120818.

22 Tran M, Tam D, Bardia A, Bhasin M, Rowe GC, Kher A, et al. PGC-1 $\alpha$ promotes recovery after acute kidney injury during systemic inflammation in mice. J Clin Invest. 2011;121: 4003-14.

23 Lynch MR, Tran MT, Ralto KM, Zsengeller ZK, Raman V, Bhasin SS, et al. TFEB-driven lysosomal biogenesis is pivotal for PGC1 $\alpha$ dependent renal stress resistance. JCI Insight. 2019;5:e1267449.

24 Drury ER, Zsengeller ZK, Stillman IE, Khankin EV, Pavlakis M, Parikh SM. Renal PGCla may be associated with recovery after delayed graft function. Nephron. 2018;138: 303-9.

25 Kelly LJ, Vicario PP, Thompson GM, Candelore MR, Doebber TW, Ventre J, et al. Peroxisome proliferator-activated receptors $\gamma$ and $\alpha$ mediate in vivo regulation of uncoupling protein (ucp-1, ucp-2, ucp-3) gene expression. Endocrinology. 1998;139:4920-7.

26 Justo R, Frontera M, Pujol E, RodríguezCuenca S, Lladó I, García-Palmer FJ, et al. Gender-related differences in morphology and thermogenic capacity of brown adipose tissue mitochondrial subpopulations. Life Sci. 2005;76:1147-58.

27 Yoshida T, Kurella M, Beato F, Min H, Ingelfinger JR, Stears RL, et al. Monitoring changes in gene expression in renal ischemia-reperfusion in the rat. Kidney Int. 2002;61:1646-54.

28 Jia P, Wu X, Pan T, Xu S, Hu J, Ding X. Uncoupling protein 1 inhibits mitochondrial reactive oxygen species generation and alleviates acute kidney injury. EBioMedicine. 2019; 49:331-40.

29 Braun N, Klumpp D, Hennenlotter J, Bedke J, Duranton C, Bleif M, et al. Ucp-3 uncoupling protein confers hypoxia resistance to renal epithelial cells and is upregulated in renal cell carcinoma. Sci Rep. 2015;5:13450. 
30 Friederich-Persson M, Persson P, Hansell P, Palm F. Deletion of uncoupling protein-2 reduces renal mitochondrial leak respiration, intrarenal hypoxia and proteinuria in a mouse model of type 1 diabetes. Acta Physiol. 2018; 223:e13058.

31 Lai EY, Luo Z, Onozato ML, Rudolph EH, Solis $\mathrm{G}$, Jose PA, et al. Effects of the antioxidant drug tempol on renal oxygenation in mice with reduced renal mass. Am J Physiol Renal Physiol. 2012;303:F64-74.
32 Qin N, Cai T, Ke Q, Yuan Q, Luo J, Mao X, et al. Ucp2-dependent improvement of mitochondrial dynamics protects against acute kidney injury. J Pathol. 2019;247:392-405.

33 Singh SP, Schragenheim J, Cao J, Falck JR, Abraham NG, Bellner L. Pgc-1 alpha regulates ho-1 expression, mitochondrial dynamics and biogenesis: role of epoxyeicosatrienoic acid. Prostaglandins Other Lipid Mediat. 2016;125:8-18.

34 Gleyzer N, Vercauteren K, Scarpulla RC. Control of mitochondrial transcription specificity factors ( $\mathrm{tfb} 1 \mathrm{~m}$ and $\mathrm{tfb} 2 \mathrm{~m}$ ) by nuclear respiratory factors (nrf- 1 and nrf-2) and pgc- 1 family coactivators. Mol Cell Biol. 2005;25:1354-66.
$35 \mathrm{Wu}$ Z, Puigserver P, Andersson U, Zhang C, Adelmant G, Mootha V, et al. Mechanisms controlling mitochondrial biogenesis and respiration through the thermogenic coactivator pgc-1. Cell. 1999;98:115-24.

36 Boddu R, Fan C, Rangarajan S, Sunil B, Bolisetty S, Curtis LM. Unique sex- and age-dependent effects in protective pathways in acute kidney injury. Am J Physiol Renal Physiol. 2017;313:F740-55. 